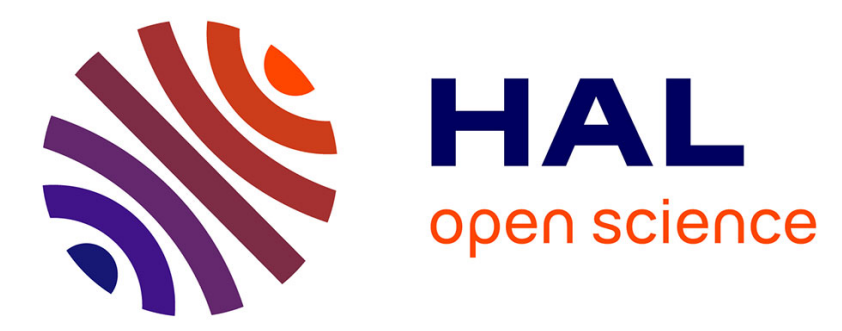

\title{
A very high cycle fatigue thermal dissipation investigation for titanium alloy TC4
}

Zhi Yong Huang, Qing Yuan Wang, Danièle Wagner, Claude Bathias

\section{To cite this version:}

Zhi Yong Huang, Qing Yuan Wang, Danièle Wagner, Claude Bathias. A very high cycle fatigue thermal dissipation investigation for titanium alloy TC4. Materials Science and Engineering: A, 2014, 600, pp.153-158. 10.1016/j.msea.2014.02.012 . hal-01421696

\section{HAL Id: hal-01421696 \\ https://hal.parisnanterre.fr/hal-01421696}

Submitted on 18 Jan 2018

HAL is a multi-disciplinary open access archive for the deposit and dissemination of scientific research documents, whether they are published or not. The documents may come from teaching and research institutions in France or abroad, or from public or private research centers.
L'archive ouverte pluridisciplinaire HAL, est destinée au dépôt et à la diffusion de documents scientifiques de niveau recherche, publiés ou non, émanant des établissements d'enseignement et de recherche français ou étrangers, des laboratoires publics ou privés. 


\title{
A very high cycle fatigue thermal dissipation investigation for titanium alloy TC4
}

\author{
Zhi Yong Huang ${ }^{\mathrm{a}, *}$, Qing Yuan Wang ${ }^{\mathrm{a}}$, Danièle Wagner ${ }^{\mathrm{b}}$, Claude Bathias ${ }^{\mathrm{b}}$ \\ a Sichuan University, School of Aeronautics and Astronautics, No. 29 Jiuyanqiao Wangjiang Road, Chengdu 610064, China \\ b Université ParisOuest Nanterre La Défense, France
}

Keywords:

Titanium alloy

VHCF

Inelastic deformation

Thermal dissipation

Fatigue dispersion

\begin{abstract}
A B S T R A C T
Titanium alloy TC4 is widely used in aeronautics applications where it is subjected to high frequency fatigue loads. Tests are performed to investigate the alloy fatigue behavior sustaining ultrasonic fatigue load in Very High Cycle Fatigue (VHCF) regime. Thermal dissipation for the alloy in $20 \mathrm{kHz}$ frequency is studied and a model is proposed to describe the temperature increment in the framework of thermodynamics by estimation of the anelastic and inelastic thermal dissipation at microscopic active sites in the reference element volume. The failure probability prediction method is used to evaluate the VHCF dispersion based on the two scale model and fatigue thermal dissipation analysis.
\end{abstract}

\section{Introduction}

With fatigue test method development, especially the application of piezoelectric fatigue test system, Very High Cycle Fatigue (VHCF) life can be obtained in a reasonable time. In High Cycle Fatigue (HCF) or VHCF, the specimen is normally loaded in the macroscopic elastic domain. Fatigue strength below the conventional fatigue limit was found and the fatigue failure still happened when the fatigue life was beyond $10^{7}$ cycles in terms of the report [1]. The crack initiation often lies in the subsurface of the specimen ("fish eye") in the VHCF regime. Through the thermal dissipation investigations [2,3], it is confirmed that the temperature rising under the load of ultrasonic frequency is induced by the anelastic and inelastic deformation at some local sites in microscopic scale.

Some authors [4] have worked on the mean fatigue limit estimation based on the temperature measurements for high cycle fatigue which consists of applying a series of stress amplitudes to obtain the average temperature increments. After a certain number of cycles loading, the evolution of temperature increase tends to be relatively stable. It is also observed that the temperature starts to increase more rapidly when the load exceeds a certain stress level. In the HCF self-heating test, the mean fatigue limit can be rapidly determined by the curve [5].

Calloch [6] has proposed a two-scale probabilistic approach to predict the fatigue life scatter in HCF based on the estimation of fatigue thermal dissipation caused by local plastic deformation.

\footnotetext{
* Corresponding author. Tel./fax: + 862885406919

E-mail address: huangzy@scu.edu.cn (Z.Y. Huang).
}

The sites like inclusions, pores, grains, grain boundaries [6,7] can be excited and have inelastic deformation under low cyclic load. With the increasing of the load and number of cycles, the quantity and volume of the sites are growing. Owing to the material heterogeneity, the local anelastic and inelastic deformation around the defects or the grains of the material are the thermal dissipation sources in the VHCF test [8].

Titanium alloy is an important metallic material with excellent mechanical response and has been widely used in aeronautical applications. Some aero-engine components like compressor blades and disks fabricated in this alloy would reduce the weight by up to $30 \%$ when compared to other alloys that have been employed for such applications. Titanium alloy is also widely used in aircraft components such as beams, joints and bulkheads, in order to undertake the loads. The high frequency of the load could lead to fatigue failure in their service period with very high cycles.

In the article, the fatigue thermal dissipation of the titanium alloy (TC4) in $20 \mathrm{kHz}$ frequency for VHCF is investigated by considering the local anelastic and inelastic deformation to estimate the temperature increment with load amplitude. Based on the investigation, the fatigue life dispersion approach is extended to VHCF regime to predict the scatter of VHCF with the help of the VHCF thermographic observation and failure probability analysis.

\section{Material}

The investigated material is a titanium alloy, TC4 (Chinese standard). The chemical composition and mechanical properties 
are shown in Tables 1 and 2, respectively. It contains 6\% Al and 4\% $\mathrm{V}$ for $\alpha$ phase and $\beta$ phase stabilization, respectively. The bar with $12 \mathrm{~mm}$ diameter was annealed at $700-800{ }^{\circ} \mathrm{C}$ and cooled in air for $1-2 \mathrm{~h}$; followed by solution in $910-940{ }^{\circ} \mathrm{C}$ and aging treatment in $520{ }^{\circ} \mathrm{C}-550{ }^{\circ} \mathrm{C}$. From the observation of the polished section in the optical microscope, two kinds of phases $\alpha, \beta$ (Fig. 1) are displayed clearly.

The ultrasonic fatigue test machine is chosen to perform the VHCF test which requires the specimen working at the resonance vibration state in $20 \mathrm{kHz}$. Hourglass shape is chosen for the specimen as shown in Fig. 2. The analytical solution for the vibration equations [1] gives out the dimensions of the specimen. The fatigue tests are performed in the machine in VHCF regime and the results are plotted in Fig. 3. It is easy to find that the fatigue strength keeps reducing with the number of cycles increasing and the fatigue life scatter is important for the titanium alloy in VHCF.

In order to obtain the temperature evolution on the specimen surface, an infrared camera is employed, whose spectral range is near the infrared domain (the wavelength is between $3.7 \mu \mathrm{m}$ and $4.8 \mu \mathrm{m}$ ). The camera is calibrated by a black body in the temperature range of $20-400{ }^{\circ} \mathrm{C}$. Same kind of specimen for the VHCF test is used to carry out the thermal dissipation experiments. They have been coated with a strongly emissive black and hightemperature resistance painting layer for limiting the errors (in this case, the emissivity coefficient is regarded as 1 ).

In the same test condition, the temperature increment depends on the load level. The temperature reaches a relative stabilization corresponding to the heat balance between the mechanical deformation dissipated energy and the thermo energy lost by the convection and radiation at the specimen surface and

Table 1

TC4 chemical composition.

\begin{tabular}{llllllll}
\hline & Al & V & Fe & C & N & H & O \\
\hline wt\% & 6 & 4 & 0.3 & 0.1 & 0.05 & 0.015 & 0.2 \\
\hline
\end{tabular}

Table 2

Mechanical properties of TC4.

\begin{tabular}{llllll}
\hline$\sigma_{b}(\mathrm{MPa})$ & $\sigma_{p 0.2}(\mathrm{MPa})$ & $E(\mathrm{GPa})$ & $\nu$ & $C_{p}\left(\mathrm{~J} /\left(\mathrm{kg}{ }^{\circ} \mathrm{C}\right)\right)$ & $\rho\left(\mathrm{kg} / \mathrm{m}^{3}\right)$ \\
\hline$\geq 900$ & $\geq 830$ & 110 & 0.34 & 0.52 & 4420 \\
\hline
\end{tabular}

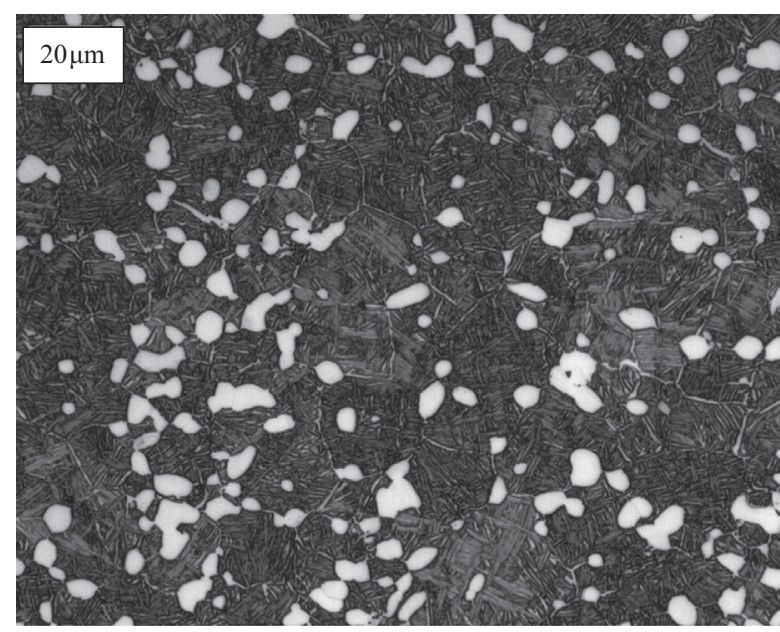

Fig. 1. TC4 microstructure.

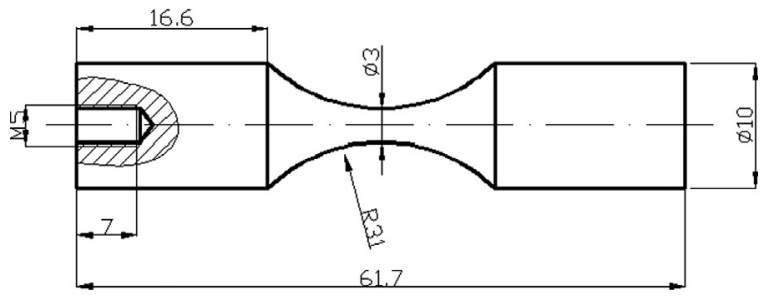

Fig. 2. VHCF specimen.

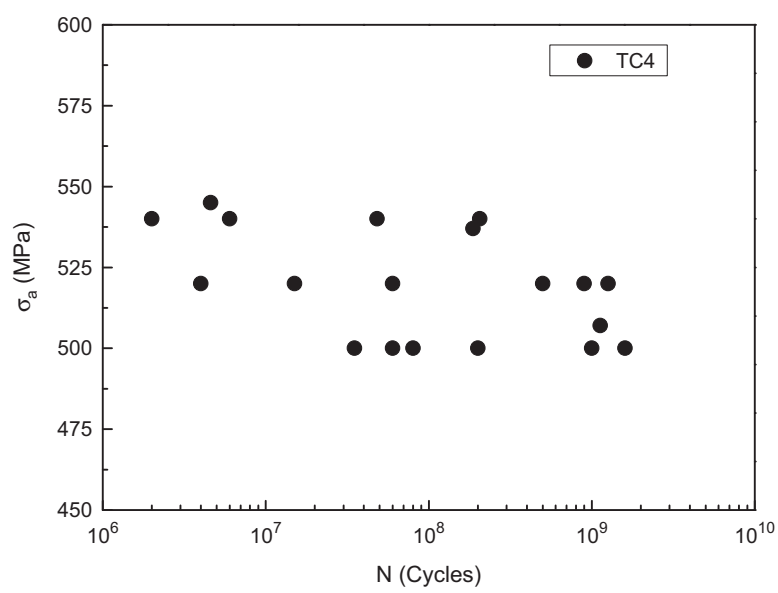

Fig. 3. VHCF test results (TC4, room temperature, $R=-1$ ).

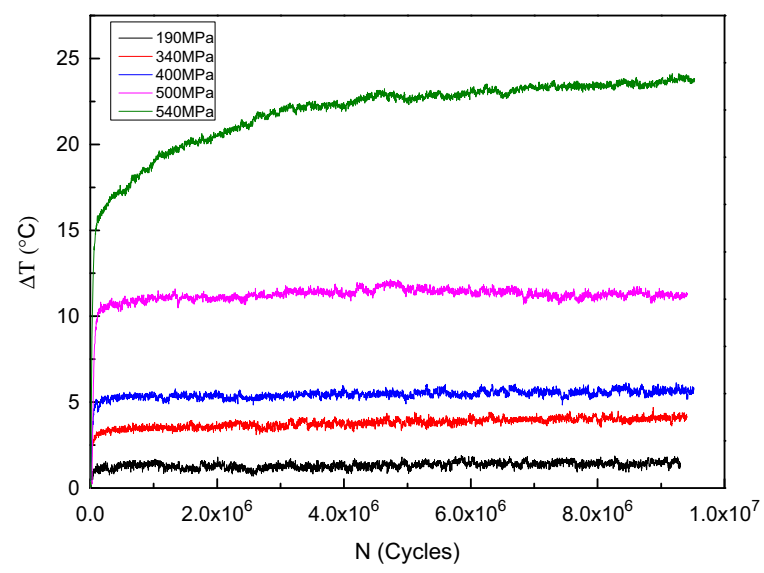

Fig. 4. Temperature evolutions at different load levels in VHCF tests.

conduction through the connection parts. The average temperature increment values (Fig. 4) recorded by the infrared camera for different stress amplitudes are plotted in Fig. 5. It comprises two relative linear curves with an "intersection point": the first one is below $450 \mathrm{MPa}$ with a slow temperature increasing slope; the second curve has a higher one. In the article, the 2 stages seem to be contributed by 2 kinds of deformation dissipations: anelastic and inelastic in VHCF which will be discussed in the next part of the article.

Fig. 6 shows the SEM observation of the broken surface of the specimen (tested with stress amplitude of $520 \mathrm{MPa}$ and failed at $9 \times 10^{8}$ cycles). The crack initiates from one of the active sites in the subsurface and forms a "fish eye" crack as shown in Fig. 6(a). In the center of the crack, a white zone (Fig. 6(b)) is observed which looks rougher than the rest of the parts where Sakai called it Fine Granular Area (FGA) [9] and it is considered as the VHCF initiation 


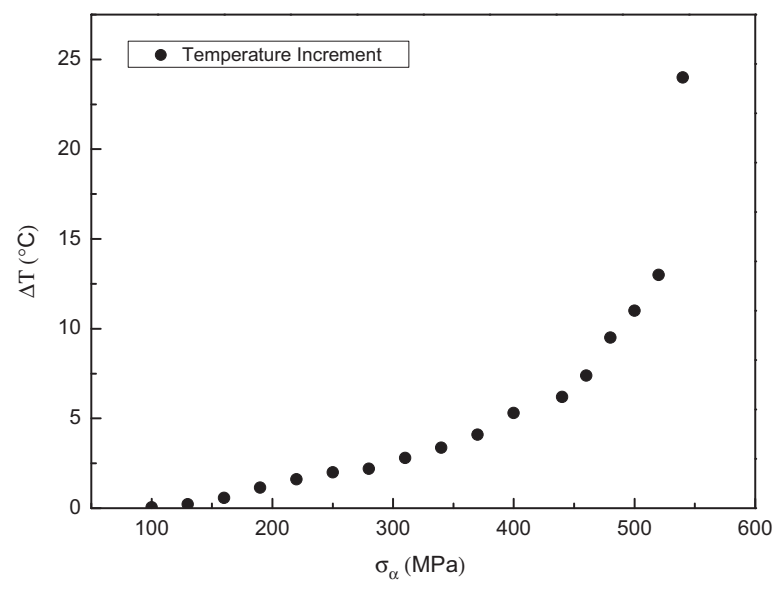

Fig. 5. Mean temperature increment with stress amplitude.

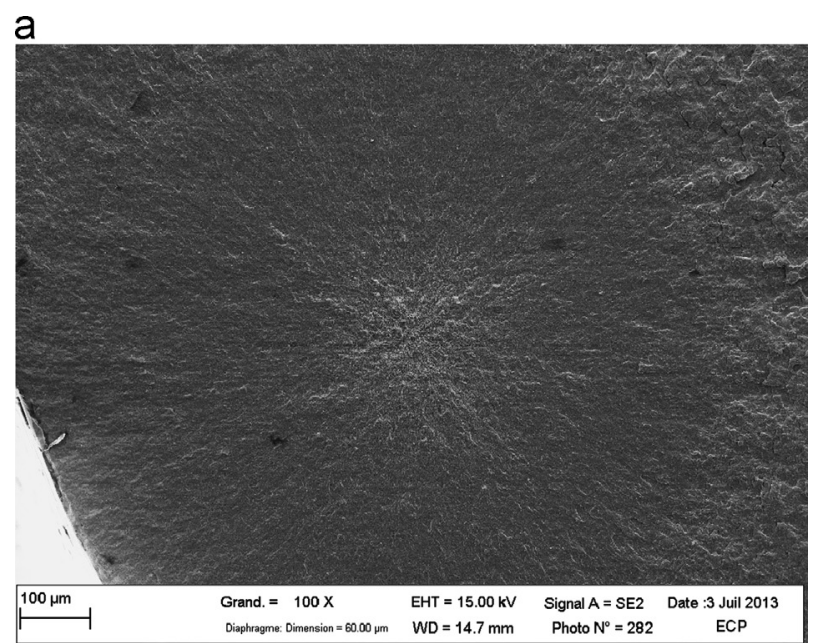

b

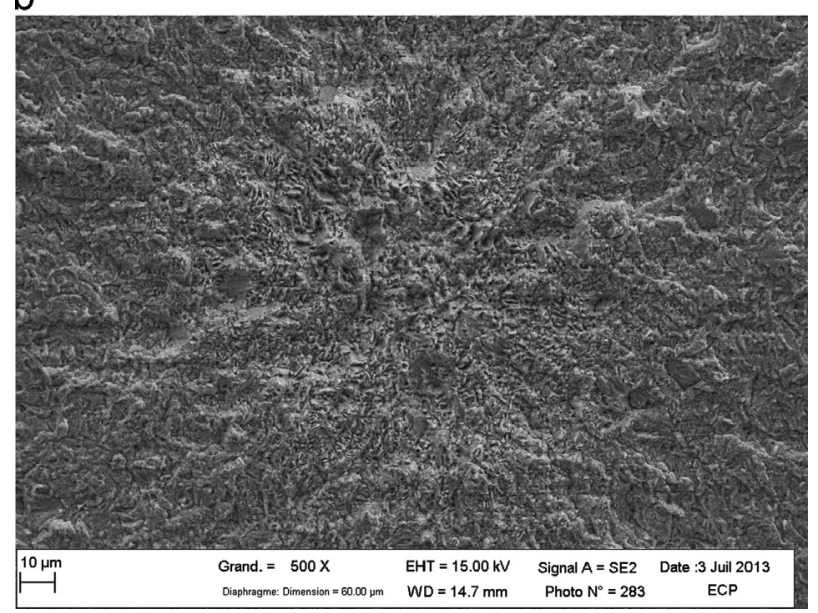

Fig. 6. Crack observation in VHCF for TC4 (a) "fish eye" crack and (b) "FGA" in the center of the crack.

zone. The grain with certain orientation or grain boundary could be the crack initiation because the inclusions have not been found in the material. The local deformation around the crack initiation site activated by the load is irreversible and could become the thermal dissipation source in the VHCF test.

\section{Very high cycle fatigue thermal dissipation approach}

The mechanism responsible for the "self-heating" in VHCF has been studied in Reference [8], which makes a consistent model to take into account the deformation in microscope. When the specimen is subjected to a cyclic load, the stress and deformation are not distributed uniformly within the specimen [10]. The heterogeneities at the microscopic scale should be considered in order to estimate the VHCF deformation and the thermal dissipation. The state of stress and deformation in the microstructure is not uniform. Thus, the polycrystalline inhomogenity is needed to be investigated and the deformation in the active sites excited by the load is considered as the source of fatigue dissipation. It is determined through the homogenization techniques based on the solution of Eshelby [11].

The average strain of a grain can be divided into three parts: elastic, anelastic and inelastic deformation and it can be expressed as follows:

$\underline{\underline{\varepsilon}}=\underline{\underline{\varepsilon}}^{e}+\underline{\underline{\varepsilon}}^{a n}+\underline{\underline{\varepsilon}}^{i n}$

\subsection{Thermodynamic frame for VHCF}

The metallic material sustaining the cyclic load, the intrinsic dissipation, is the consequence of 2 kinds of deformation: anelastic and inelastic induced by the dislocation movements. The concepts of continuum thermodynamics are used to analyze the temperature measurements. At each instant $t$, the thermodynamic state of each site is characterized by the state variables $[4,6]$.

$\psi=\psi_{\text {mat }}\left(1-f_{v}\right)+\psi_{\text {in }} f_{v}$

where $f_{v}=V_{0} / V_{\Omega}$ is the inelastic deformation volume fraction in $V_{\Omega}$. $V_{0}$ is the sum of the grains having the inelastic deformation. If the local stress is over the microscopic yield stress in the grain, the inelastic deformation appears and its volume needs to be counted into the volume $V_{0}$.

$\psi_{\text {mat }}$ and $\psi_{\text {in }}$ are the free energy densities of the matrix and the active sites.

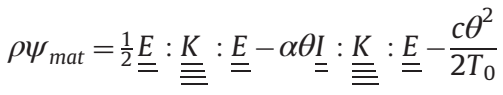

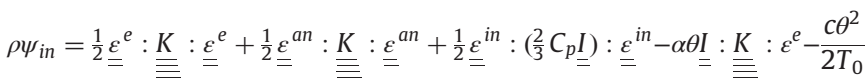

where $\underline{K}$ is the elastic tensor; $\rho$ is the mass density; $C_{p}$ is the 三

specific heat capacity; $I$ is the fourth order unit tensor; and $\alpha$ is the coefficient of thermal expansion.

By application of the first law of thermodynamics, the external power $\Pi$ can be written as

$\Pi=\left(1-f_{v}\right) \underline{\underline{\Sigma}}: \underline{\underline{\dot{E}}}+f_{v} \underline{\underline{\sigma}}: \underline{\underline{\dot{\varepsilon}}}^{e}+f_{v} \underline{\underline{\sigma}}: \underline{\underline{\dot{\varepsilon}}}^{a n}+f_{v}(\underline{\underline{\sigma}}-\underline{\underline{x}}): \underline{\underline{\dot{\varepsilon}}}^{i n}+f_{v} \underline{\underline{x}}: \underline{\underline{\dot{\varepsilon}}}^{i n}$

where $\left(1-f_{v}\right) \underline{=}: \underline{\underline{\dot{E}}}$ and $f_{v} \underline{\underline{\sigma}}: \underline{\underline{\dot{\varepsilon}}}^{e}$ are the recoverable elastic power; $f_{\nu} \underline{\underline{\sigma}}: \underline{\underline{\varepsilon}}^{a n}$ is the anelastic deformation power; and $f_{v}(\underline{\underline{\sigma}}-\underline{\underline{x}}): \underline{\underline{\dot{\varepsilon}}}^{\text {in }}$ is the inelastic power. The dissipated power includes 2 parts: anelastic dissipation and inelastic dissipation.

\subsection{Anelastic deformation in $\mathrm{VHCF}$}

The dislocations move slightly without detaching the points of anchoring under the low amplitude load in anelastic deformation. The friction of the dislocation could be considered as the source of 
low thermal dissipation, but the deformation is reversible since the linear tension/compression is a restoring force bringing the dislocations to an equilibrium position. The anelastic deformation is more difficult to be measured because it does not result in any microstructural change [12].

A Kelvin-Voigt type element is used to describe the stress and the anelastic deformation relation. The variables include the viscosity characteristics of the deformation through the viscosity damp while considering its reversibility character by the spring stiffness:

$\underline{\underline{\sigma}}=\mu \underline{\underline{\varepsilon}}^{a n}+\dot{\eta} \underline{\underline{\varepsilon}}^{a n}$

where $\eta$ is the viscosity of the material and $\mu$ is the elastic shear modulus. In the case of very low cyclic load, few activated sites have inelastic deformation. For simplification, the hypothesis is made that the local stress would be regarded as $=\Sigma$ in low cyclic load case, and $\Sigma$ could be written as

$\underline{\underline{\Sigma}}=\underline{\underline{\Sigma}}_{m}+\underline{\underline{\Sigma}}_{a} \sin \left(2 \pi f_{r} t\right)$

where $f_{r}$ is the loading frequency. The average thermal dissipation per cycle is obtained in a period $T_{S}$ due to the anelastic deformation and is given out as follows:

$D_{a n}=\frac{1}{T_{s}} \int_{t}^{t+T_{s}} \underline{\underline{\sigma}}: \underline{\underline{\varepsilon}}^{a n} d t$

In terms of Eqs. (6) and (7), the anelastic deformation could be obtained by solving the differential equation. The anelastic dissipation per cycle in this situation at the low stress amplitude case can be obtained by Eq. (8) and rewritten as

$D_{a n}=\frac{2 \eta\left(\pi f_{r} J_{2}(\underline{\bar{\Sigma}})\right)^{2}}{\mu^{2}+4 \pi^{2} f_{r}^{2} \eta^{2}}$

where $\mu=E / 2(1+\nu)$.

\subsection{Inelastic deformation in $\mathrm{VHCF}$}

Inelastic deformation is from irreversible dislocations slip caused by the local inhomogeneous stress, which can give rise to a higher thermal dissipation. In terms of the theory of linear kinematic hardening [13], the back stress in active sites is expressed as

$\underline{\dot{x}}=\frac{2}{3} C \underline{\dot{\varepsilon}}^{\text {in }}$

$\underline{\underline{\varepsilon}}^{i n}=\dot{\lambda}_{i n} \frac{\partial f}{\partial s}$

$f=J_{2}(\underline{\underline{s}}-\underline{\underline{x}})-\sigma_{y}^{\mu}$

where $\dot{\varepsilon}^{\text {in }}$ is assumed to be local inelastic deformation in the active sites; $f$ is the yield surface and $C$ is the material hardening coefficient.

$\underline{\underline{s}}=\underline{\underline{\sigma}}-\frac{1}{3} \operatorname{trace}(\underline{\underline{\sigma}}) \underline{\underline{I}}$

$J_{2}(\underline{\underline{s}}-\chi)=\sqrt{\frac{3}{2}(\underline{\underline{s}}-\underline{\underline{x}}):(\underline{\underline{s}}-\underline{\underline{x}})}$

where $\underline{\underline{s}}$ is the local deviator stress tensor. The plastic multiplier $\dot{\lambda}_{\text {in }}$ can be obtained from the consistency condition:

$\dot{f}=\frac{\partial f}{\partial \underline{\underline{\sigma}}}: \underline{\underline{\sigma}}+\frac{\partial f}{\partial \underline{\underline{x}}}: \underline{\underline{x}}$

The microscopic stress is evaluated from the macroscopic stress by the law of localization which is obtained by a homogenization procedure [11]:

$\underline{\underline{\sigma}}=\underline{\underline{\Sigma}}-2 \mu(1-\beta) \underline{\underline{\varepsilon}}^{i n}$

where $\beta=2(4-5 \nu) / 15(1-\nu)$, given by the Eshelby two-scale analysis of a spherical inclusion; $\mu$ and $\nu$ are the shear modulus and the Poisson's ratio of the material, respectively.

By using the localization and the kinematic hardening laws, the above Eq. (15) can be rewritten as

$\frac{\partial f}{\partial \sigma}:\left[\underline{\underline{\Sigma}}-2 \mu(1-\beta) \underline{\underline{\dot{\varepsilon}}}^{\text {in }}-\frac{2}{3} C \underline{\underline{\varepsilon}}^{i n}\right]=0$

Consequently, one can deduce the following relation by using the normality rule with the hardening modulus: $h=C+3 \mu(1-\beta)$. The derivation is listed as below:

$\underline{\underline{\varepsilon}}^{i n}=\dot{\lambda}_{i n} \frac{\partial f}{\partial \underline{\underline{S}}}=\dot{\lambda}_{\text {in }} \frac{\partial f}{\partial \underline{\underline{\sigma}}}$

Replacing $\underline{\dot{\varepsilon}}^{\text {in }}$ in Eq. (18), the plastic multiplier $\dot{\lambda}_{\text {in }}$ is rewritten as

$\dot{\lambda}_{\text {in }}=\frac{H(f)}{h} \frac{\partial f}{\partial \underline{\underline{\sigma}}}: \underline{\dot{\Sigma}}$

where $H(f)$ is the Heaviside step function (i.e., $H(f)=0$, if $f<0$ and $H(f)=1$ if $f>=0$ ). The dissipated energy density $d_{1}$ becomes

$d_{1}=f_{v}(\underline{\underline{\sigma}}-\underline{\underline{X}}): \underline{\underline{\varepsilon}}^{\text {in }}$

Integrating Eq. (20) by introducing Eqs. (19) and (20) in a loading cycle:

$d_{\text {in }}=\int_{\text {cycle }} d_{1} d t$

Considering $J_{2}(\underline{\underline{\sigma}}-\underline{\underline{X}})=\sigma_{y}$ when $f \geq 0$, above Eq.(21) yields:

$d_{i n}=4 \int_{\sigma_{y}}^{\Sigma_{a}} f_{v} \frac{H(f)}{h} \sigma_{y} d J_{2}(\underline{\underline{\Sigma}})=4 f_{v} \frac{1}{h} \sigma_{y}\left\langle\Sigma_{a}-\sigma_{y}\right\rangle$

where \langle\rangle are Macauley brackets.

\subsection{Probabilities model and thermal dissipation estimation in VHCF}

The microscopic yield stress is considered as a probabilistic variable. The probability for finding $k$ active sites in a domain $\Omega$ of volume $V_{\Omega}$ follows Poisson distribution:

$P_{k}\left(V_{\Omega}\right)=\exp \left(-\lambda V_{\Omega}\right) \frac{\left(\lambda V_{\Omega}\right)^{k}}{k !}$

where $V_{\Omega}$ means the volume in the domain $\Omega$. $\lambda$ is the intensity of activated sites and assumed to be a function of the macroscopic equivalent stress amplitude $\Sigma$ in uniaxial tension-compression fatigue load for a smooth specimen [6].

$\lambda(\Sigma)=\frac{1}{V_{0}}\left(\frac{\Sigma}{S_{0}}\right)^{m}$

where $S_{0}$ and $m$ are two parameters depending on the material and $V_{0}$ is the referring volume. The average number of active sites can be calculated as $\lambda(\Sigma) V_{\Omega}$ in a domain $\Omega$ with volume $V_{\Omega}$.

The failure probability $P_{F}$ in the domain under homogeneous stress $\Sigma$ having $\lambda V_{\Omega}$ active sites equals the probability of finding at least 1 active site in the volume $V_{\Omega}$, which could induce the fatigue failure. The Weibull model can be regained within the framework of Poisson point process [14].

$P_{F}=P_{k \geq 0}\left(V_{\Omega}\right)=1-P_{k=0}\left(V_{\Omega}\right)=1-\exp \left(-\lambda V_{\Omega}\right)$ 
$P_{F}=1-\exp \left[-\frac{V_{\Omega}}{V_{0}}\left(\frac{\Sigma}{S_{0}}\right)^{m}\right]$

If the stress field is no longer homogeneous, the previous result can be generalized and the approach can be extended to the VHCF regime [8].

The HCF life prediction is often characterized by the assumption of "fatigue limit". However in VHCF, where lifetimes exceed $10^{9}$ cycles the fatigue strength decreases continuously with an increasing number of cycles and the traditionally understood "fatigue limit" is not typically observed [1]. Since the fatigue strength decreases continuously with increasing number of cycles, it seems to be hard to find the 'fatigue limit' in VHCF in terms of the investigation of Bathias [1]. The fatigue strength at $10^{9}$ cycles with $50 \%$ failure probability is proposed to replace the "fatigue limit" in this article.

In a domain $\Omega, N(\Omega, \Sigma)$ is the number of active sites in a onedimension load condition applied on a smooth specimen at a stress amplitude $\Sigma$ and the $N(\Omega, \Sigma+d \Sigma)$ is the number of sites for a stress amplitude increment $\Sigma+d \Sigma$.

The number of sites with yield stress between $\Sigma$ and $\Sigma+d \Sigma$ in a volume $V$,

$d N_{a}(\Sigma)=V \lambda(\Sigma+d \Sigma)-V \lambda(\Sigma)=V \frac{d \lambda}{d \Sigma} d \Sigma$

In the domain $V_{\Omega}$, the total cyclic dissipation equals the summation of inelastic deformation energy associated with different inclusions' yield stress $\Sigma$ weighted by the number of sites having this yield.

$D_{\text {in }}=\int_{0}^{\Sigma_{a}} V_{\Omega} d_{i n} \frac{d \lambda}{d \Sigma} d \Sigma$

Integrating Eq. (28), the density of cyclic dissipation at the amplitude of stress $\Sigma_{a}$ :

$D_{\text {in }}=\frac{4 m \Sigma_{a}^{2}}{h(m)(m+2)}\left(\frac{\Sigma_{a}}{S_{0}}\right)^{m}$

The total dissipation per cycle of VHCF is the sum of anelastic and inelastic thermal dissipations and is expressed as follows:

$D=D_{a n}+D_{\text {in }}$

$D=V_{0} \frac{2 \eta\left(\pi f_{r} \Sigma_{a}\right)^{2}}{\mu^{2}+4 \pi^{2} f_{r}^{2} \eta^{2}}+\frac{4 m \Sigma_{a}^{2}}{h(m)(m+2)}\left(\frac{\Sigma_{a}}{S_{0}}\right)^{m}$

The dissipated energy leading to the temperature increment can be evaluated by the heat conduction equation [15]:

$\dot{\theta}+\frac{\bar{\theta}}{\tau_{e q}}=\frac{f_{r} D}{\rho C_{p}}$

The average temperature increment which is a function of stress amplitude is estimated as

$\bar{\theta}=\frac{f_{r} \tau_{e q}}{\rho C_{p}}\left[\frac{2 \eta\left(\pi f_{r} \Sigma_{a}\right)^{2}}{\mu^{2}+4 \pi^{2} f_{r}^{2} \eta^{2}}+\frac{4 m \Sigma_{a}^{2}}{h(m)(m+2)}\left(\frac{\Sigma_{a}}{S_{0}}\right)^{m}\right]$

Eq. (33) shows that the evolution of mean stability temperature depends on 3 parameters, namely, $\tau_{e q}$ (the equivalent conduction time), $S_{0}$ (reference stress) and $m$ (shape parameter of Weibull distribution) [16].
The $S-N$ curve of VHCF in uniaxial test condition can be described by the Basquin model [17] with a "pseudo-fatigue limit" [18]. The fatigue strength at $10^{9}$ or $10^{10}$ cycles with certain probability $P_{F}$ could be used to replace the "fatigue limit":

$N_{f}\left(\Sigma_{a}, P_{F}\right)=\left(\frac{\Sigma_{a}-\Sigma_{D}\left(P_{F}\right)}{M}\right)^{-\gamma}$

where $\Sigma_{D}\left(P_{F}\right)$ is the fatigue strength at $10^{9}$ cycles and $P_{F}$ is the fatigue failure possibility. The parameter $M$ and exponent $\gamma$ are both Basquin parameters determined by the VHCF tests. Considering the dispersion of VHCF test results due to the random appearance of the microscopic inelastic deformation, the fatigue strength with failure probability can be rewritten as follows by referring to Eq. (26):

$\Sigma_{D}\left(P_{F}\right)=\Sigma_{D}(0.5)\left[\frac{\ln \left(1-P_{F}\right)}{\ln (1-0.5)}\right]^{\frac{1}{m}}$

The fatigue strength at $10^{9}$ cycles with different failure possibilities can be deduced from Eq. (35). The predicted fatigue strength only depends on the shape parameter $m$ of Weibull distribution if the mean fatigue strength has been determined with $50 \%$ probability from the $S-N$ curve up to $10^{9}$ cycles.

\section{Identification and validation}

The VHCF temperature increment is attributed to the microscopic anelastic and inelastic dissipation with high frequency load. The thermal dissipation part caused by the local anelasctic deformation is estimated by the first term of Eq. (33). The viscosity of elastic $(\eta)$ is the result of the out phase of the local stress and strain at the high frequency [10] and it could be determined by the first temperature increment stage in Fig. 5.

The inelastic deformation could accelerate the temperature increment more significantly at the second temperature rising stage (Fig. 5). The parameter $h$ simultaneously appears in the model and plays a role of scale; $\tau_{e q}$ is the equivalent time depending on the heat transfer boundary conditions of the

Table 3

Parameters for the thermal dissipation and fatigue scatter model.

\begin{tabular}{lllllll}
\hline$m$ & $M(\mathrm{MPa})$ & $\gamma$ & $\sigma_{D}(\mathrm{MPa})$ & $\eta(\mathrm{MPa})$ & $S_{0}(\mathrm{MPa})$ & $\tau_{\text {eq }}(\mathrm{s})$ \\
\hline 21 & 290 & 7.35 & 490 & $5.3 \times 10^{6}$ & 670 & 40 \\
\hline
\end{tabular}

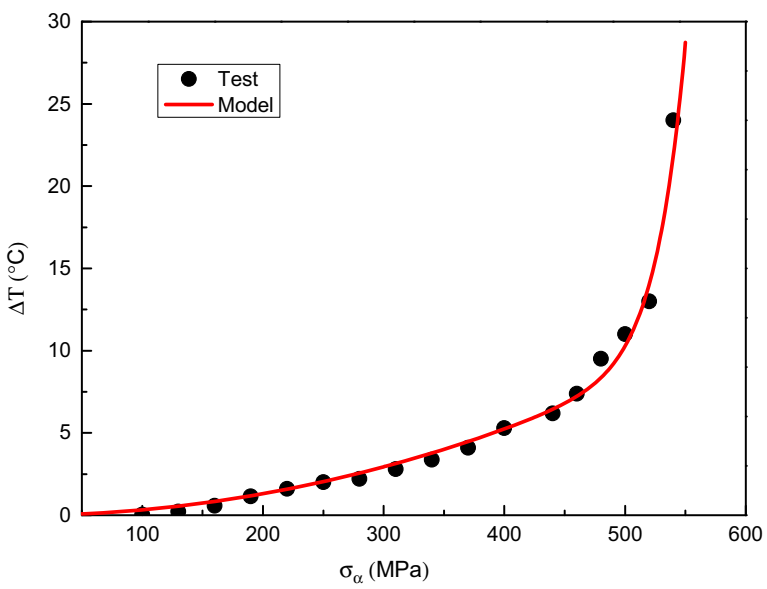

Fig. 7. Estimation of the temperature increment self-heating evolution versus stress amplitude in ultrasonic fatigue. 


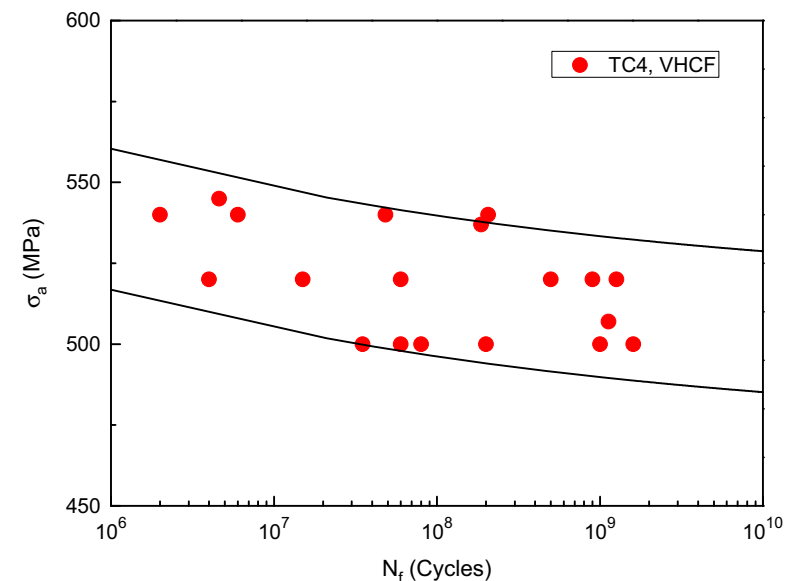

Fig. 8. Prediction of VHCF scatter for TC4 alloy for $20 \%$ and $80 \%$ failure probability.

specimen and can be determined as 40 by the experimental temperature drop curve after unloading the VHCF test; the $m$, which is more or less relayed on the evolution of temperature, identified directly as 21 with the help of the self heating test (Figs. 4 and 5). The $S / N$ curve described by the Basqin model with the parameters can be determined by the VHCF test results in Fig. 3. The model with considering the dispersion of the tests for VHCF is also due to the randomness appearance of microscopic inelastic deformation. The parameters of the model (Eq. (32)) are listed in Table 3. The VHCF strength dispersion with 20\% and $80 \%$ failure probability is estimated and plotted in Fig. 8. The model estimation is given in Fig. 7 which seems to be good to describe the temperature increment at high load frequency $(20 \mathrm{kHz})$ in VHCF and the scatter of VHCF seems to be well predicted.

\section{Conclusions}

The paper investigates the VHCF scatter and thermal dissipation under the high frequency load for TC4 alloy. The conclusions are listed as follows:

The fatigue strength of the TC4 alloy is decreased with the number of cycles increasing and the "FGA" is found in the center of the "fish eye" crack in the TC4 VHCF test by SEM fracture surface analysis.

The VHCF thermal dissipation estimation is proposed to describe the mean temperature increment with stress amplitude in the thermodynamic framework by the evaluation of the anelastic and inelastic deformation dissipations based on the two-scale method.

The probability model for VHCF scatter improved from the Basquin model with the help of the fatigue thermal dissipation estimation seems to be able to predict the fatigue dispersion.

\section{Acknowledgment}

The authors thank National Natural Science Foundation of China for providing financial support (Nos. 51101107 and 11372201).

\section{References}

[1] C. Bathias, P.C. Paris, Gigacycle Fatigue in Mechanical Practice, Marcel Dekker, New York, 2005

[2] N. Ranc, D. Wagner, P.C. Paris, Acta Mater. 56 (2008) 4012-4021.

[3] E. Charkaluk, A. Constantinescu, Mech. Mater. 41 (2009) 483-494.

[4] M.P. Luong, Mech. Mater. 28 (1998) 155-163.

[5] F. Curà, G. Cuti, R. Sesana, Int. J. Fatigue 27 (2005) 453-459.

[6] C. Doudard, S. Calloch, P. Cugy, A. Galtier, F. Hild, Fatigue Fract. Eng. Mater. Struct. 28 (2005) 279-288.

[7] H. Sabar, M. Berveiller, V. Favier, S. Berbenni, Int. J. Solids Struct. 39 (2002) 3257-3276.

[8] Z.Y. Huang, Q.Y. Wang, D. Wagner, C. Bathias, J.L. Chaboche, Fatigue Fract. Eng. Mater. Struct. 36 (2013) 462-468.

[9] T. Sakai, in: J.E. Allison, J.W. Jones, J.M. Larsen, R.O. Ritchie (Eds.), Proceedings on Very High Cycle Fatigue 4, The Minerals, Metals and Materials Society, Warrendale, PA, 2007, pp. 3-12.

[10] A. Paquin, S. Berbenni, V. Favier, X. Lemoine, M. Berveiller, Int. J. Plast. 17 (2001) 1267-1302.

[11] J.D. Eshelby, Proc. R. Soc. Lond. A 241 (1957) 376-396.

[12] C. Mareau, Modélisation de l'échauffement et de la microplasticité des aciers sous sollicitations cycliques (Ph.D. thesis), ENSAM, 2007.

[13] J. Lemaitre, J.L. Chaboche, Mechanics of Solid Materials, Cambridge University Press, Cambridge, 1990.

[14] A. Ezanno, C. Doudard, S. Calloch, T. Millot, J.-L. Heuzé, Proc. Eng. 2 (2010) 967-976.

[15] T. Boulanger, A. Chrysochoos, C. Mabru, A. Galtier, Int. J. Fatigue 26 (2004) 221-229.

[16] W. Weibull, ASME. J. Appl. Mech. 18 (1951) 293-297.

[17] O.H. Basquin, Proc. Am. Soc. Test. Mater. 10 (1910) 625-630.

[18] J.L. Chaboche, L.M. Kaminski, P. Kanoute, Dtsch. Verb. Materialforschung Prufung e.V. (2009) 627-639. 